\title{
Can histopathologists reliably diagnose molar pregnancy?
}

\author{
A J Howat, S Beck, H Fox, S C Harris, A S Hill, C M Nicholson, R A Williams
}

Department of

Histopathology, Royal

Preston Hospital,

Preston PR2 4HG

A J Howat

C M Nicholson

Department of

Histopathology,

Doncaster Royal

Infirmary

$S$ Beck

Department of

Pathological Sciences,

University of

Manchester

H Fox

Department of

Histopathology,

Staffordshire General

Infirmary

S C Harris

Department of

Histopathology, Jessop

Hospital for Women,

Sheffield

A S Hill

Department of

Pathology,

Wangaratta District

Base Hospital,

Wangaratta, Victoria,

Australia

R A Williams

Correspondence to:

A J Howat

Accepted for publication

9 February 1993

\begin{abstract}
Aims-To assess the degree of difficulty in diagnosing partial mole by analysing intraobserver and interobserver agreement among a group of pathologists for these diagnoses.

Methods-Fifty mixed cases of partial mole, complete mole, and non-molar pregnancy were submitted to seven histopathologists, two of whom are expert gynaecological pathologists; the other five were district general hospital consultants, one of whom works in Australia. These participants gave each slide a firm diagnosis of either partial mole, complete mole, or non-molar pregnancy. Some 12 months later, the slides were recoded and again submitted for a second diagnostic round to assess intraobserver as well as interobserver agreement. Standard histological criteria for each diagnostic category were circulated with the slides.

Results- $\kappa$ statistics showed that complete mole could be reliably distinguished from non-molar pregnancy, but neither non-molar pregnancy nor complete mole could be easily differentiated from partial mole. In only 35 out of $\mathbf{5 0}$ cases was there agreement between five or more of the seven participants. Agreement between the expert gynaecological pathologists was no better than for others in the group. Interestingly, the intraobserver agreement for each pathologist was good to excellent.

Conclusions-These results imply that the reported histological criteria are either not being applied consistently or that they are lacking in practical use. An atypical growth pattern of trophoblast, rather than the polar accentuation seen in normal first trimester pregnancies, seems to be the important diagnostic histological feature for partial mole. Ploidy studies might also help with problem cases.
\end{abstract}

\section{$(\Im$ Clin Pathol 1993;46:599-602)}

In daily practice one recurring problem for histopathologists is whether products of conception show molar features or merely hydropic change associated with fetal death..$^{12}$ This is especially so for partial moles which may have fetal parts and membranes as well as villi, trophoblast, and decidua. There are, however, histological criteria that are said to easily distinguish between complete mole, non-molar pregnancy, and partial mole. ${ }^{1-4}$ The diagnosis of partial mole or complete mole is important, with the patient having to enter the follow up surveillance programme for persistent trophoblastic disease and a request for her not to become pregnant; this entails measurement of urinary $\beta$ human chorionic gonadotrophin for six to 12 months. ${ }^{56}$

This study was designed to test how good histopathologists are at differentiating complete mole, partial mole, and non-molar pregnancy, to assess the value of the recognised histological criteria.

\section{Methods}

Fifty mixed cases of non-molar pregnancy, partial mole, and complete mole were selected from the files at Royal Preston Hospital and the Jessop Hospital for Women. Slides were coded and submitted to the seven participants. Some 12 months later, the slides were recoded and submitted for a second round. Table 1 shows the histological criteria sent with the slides. Ploidy studies were not carried out on these cases.

The results were then statistically evaluated for intra- and interobserver agreement as follows:

\section{Table 1 Histological criteria}

\begin{tabular}{l} 
Non-molar \\
Grossly normal/few vesicles \\
Often fetus/fetal parts \\
Variable hydropic change \\
Atrophic artenuated trophoblast \\
Occasional syncytial sprouts \\
Partial mole \\
Normal volume of placenta \\
Often fetus/fetal parts \\
Small vesicles mixed with normal villi \\
Variable hydropic change \\
Variable trophoblast hyperplasia \\
Circumferential proliferation \\
Central cisternal degeneration \\
Scalloping of villi with trophoblast "inclusions" \\
Some villi more normal with blood vessels \\
Some small fibrosed avascular villi \\
Complete mole \\
Bulky uterus > dates \\
Bunch of grapes grossly \\
Rarely fetal tissue (if ever) \\
Swollen avascular villi \\
Variable trophoblast hyperplasia \\
\hline
\end{tabular}


Table 2 Histological opinions

\begin{tabular}{|c|c|c|c|c|c|c|c|c|c|c|c|c|c|c|c|}
\hline \multirow[b]{3}{*}{ Slide No } & \multirow[b]{3}{*}{ Run } & \multicolumn{14}{|c|}{ Pathologist } \\
\hline & & \multicolumn{2}{|l|}{$A$} & \multicolumn{2}{|l|}{$B$} & \multicolumn{2}{|l|}{$C$} & \multicolumn{2}{|l|}{$D$} & \multicolumn{2}{|l|}{$E$} & \multicolumn{2}{|l|}{$F$} & \multicolumn{2}{|l|}{$G$} \\
\hline & & 1 & 2 & 1 & 2 & 1 & 2 & 1 & 2 & 1 & 2 & 1 & 2 & 1 & 2 \\
\hline 1 & & NM & NM & NM & PM & PM & PM & PM & NM & PM & PM & PM & NM & NM & PM \\
\hline 2 & & NM & NM & NM & NM & NM & NM & NM & PM & NM & NM & PM & PM & NM & NM \\
\hline 3 & & NM & NM & PM & PM & PM & PM & PM & PM & NM & NM & CM & PM & NM & NM \\
\hline 4 & & NM & NM & PM & NM & NM & NM & NM & NM & NM & NM & NM & NM & NM & NM \\
\hline 5 & & $\mathrm{NM}$ & PM & NM & PM & NM & NM & NM & NM & NM & NM & NM & NM & NM & PM \\
\hline 6 & & NM & NM & NM & NM & NM & NM & NM & NM & NM & NM & NM & NM & NM & NM \\
\hline 7 & & $\mathrm{CM}$ & $\mathrm{CM}$ & PM & CM & $\mathrm{CM}$ & CM & $\mathrm{CM}$ & $\mathrm{CM}$ & $\mathrm{CM}$ & $\mathrm{CM}$ & CM & CM & PM & PM \\
\hline 8 & & NM & NM & NM & NM & NM & NM & NM & NM & NM & NM & NM & NM & NM & NM \\
\hline 9 & & PM & PM & PM & PM & NM & NM & PM & NM & PM & PM & PM & NM & PM & PM \\
\hline 10 & & $\mathrm{PM}$ & PM & PM & $\mathrm{PM}$ & PM & PM & PM & PM & PM & $\mathrm{CM}$ & $\mathrm{CM}$ & PM & PM & PM \\
\hline 11 & & CM & CM & CM & CM & CM & CM & $\mathrm{CM}$ & PM & $\mathrm{CM}$ & $\mathrm{CM}$ & $\mathrm{CM}$ & $\mathrm{CM}$ & $\mathrm{PM}$ & PM \\
\hline 12 & & NM & NM & PM & PM & NM & NM & NM & PM & NM & NM & NM & NM & PM & PM \\
\hline 13 & & NM & NM & NM & NM & NM & NM & NM & NM & NM & NM & NM & NM & NM & NM \\
\hline 14 & & NM & NM & NM & NM & NM & PM & NM & NM & NM & NM & PM & NM & NM & NM \\
\hline 15 & & PM & PM & PM & $\mathbf{P M}$ & NM & PM & PM & PM & PM & PM & PM & $\mathbf{P M}$ & PM & PM \\
\hline 16 & & NM & PM & PM & $\mathbf{P M}$ & NM & NM & NM & NM & NM & PM & NM & NM & PM & NM \\
\hline 17 & & $\mathrm{CM}$ & $\mathrm{CM}$ & PM & CM & $\mathrm{CM}$ & $\mathrm{CM}$ & $\mathrm{CM}$ & PM & CM & $\mathrm{CM}$ & $\mathrm{CM}$ & $\mathrm{CM}$ & $\mathrm{CM}$ & $\mathrm{CM}$ \\
\hline 18 & & $\mathbf{P M}$ & $\mathrm{CM}$ & CM & $\overline{C M}$ & PM & $\overline{\mathrm{CM}}$ & PM & $\mathrm{PM}$ & PM & $\mathrm{CM}$ & $\widehat{C M}$ & $\mathrm{CM}$ & PM & $\mathbf{P M}$ \\
\hline 19 & & $\mathrm{CM}$ & $\mathrm{CM}$ & $\mathbf{C M}$ & $\mathrm{CM}$ & $\mathrm{CM}$ & $\mathrm{CM}$ & $\mathrm{CM}$ & CM & $\mathbf{C M}$ & $\mathrm{CM}$ & $\mathrm{CM}$ & CM & CM & PM \\
\hline 20 & & NM & NM & NM & PM & NM & NM & NM & PM & PM & PM & NM & PM & PM & PM \\
\hline 21 & & PM & PM & NM & PM & NM & NM & NM & PM & NM & NM & PM & PM & PM & PM \\
\hline 22 & & NM & NM & NM & NM & NM & PM & NM & NM & NM & NM & NM & NM & NM & NM \\
\hline 23 & & CM & $\mathrm{CM}$ & CM & CM & $\mathrm{CM}$ & $\mathrm{CM}$ & $\mathrm{CM}$ & $\mathrm{CM}$ & CM & PM & PM & CM & $\mathrm{CM}$ & PM \\
\hline 24 & & NM & NM & NM & NM & NM & NM & NM & NM & NM & NM & PM & PM & NM & NM \\
\hline 25 & & NM & NM & NM & NM & NM & NM & NM & NM & NM & NM & NM & NM & NM & NM \\
\hline 26 & & NM & NM & NM & PM & NM & NM & NM & NM & PM & PM & NM & NM & PM & PM \\
\hline 27 & & $\mathrm{CM}$ & CM & CM & $\mathrm{CM}$ & $\mathrm{CM}$ & CM & CM & $\mathrm{CM}$ & $\mathrm{CM}$ & $\mathrm{CM}$ & $\mathrm{CM}$ & $\mathrm{CM}$ & $\mathrm{CM}$ & $\mathrm{CM}$ \\
\hline 28 & & NM & NM & NM & NM & PM & PM & PM & PM & NM & NM & PM & PM & PM & PM \\
\hline 29 & & PM & NM & PM & PM & NM & NM & PM & PM & PM & PM & PM & PM & NM & PM \\
\hline 30 & & CM & CM & CM & CM & CM & CM & $\mathrm{CM}$ & $\mathrm{CM}$ & CM & CM & CM & CM & PM & PM \\
\hline 31 & & $\mathrm{CM}$ & $\mathrm{CM}$ & $\mathrm{CM}$ & $\mathrm{CM}$ & $\mathrm{CM}$ & $\mathrm{CM}$ & $\mathrm{CM}$ & PM & $\mathbf{P M}$ & $\mathrm{CM}$ & $\mathrm{CM}$ & $\mathrm{CM}$ & $\mathrm{CM}$ & PM \\
\hline 32 & & NM & NM & NM & NM & NM & NM & NM & NM & NM & $\mathrm{PM}$ & NM & NM & NM & NM \\
\hline 33 & & NM & $\mathrm{PM}$ & NM & $\mathbf{P M}$ & NM & NM & PM & NM & NM & $\mathbf{P M}$ & NM & NM & PM & PM \\
\hline 34 & & CM & $\mathrm{CM}$ & PM & CM & $\mathrm{CM}$ & PM & $\mathrm{CM}$ & $\mathrm{CM}$ & $\mathbf{C M}$ & CM & CM & CM & PM & $\mathrm{CM}$ \\
\hline 35 & & NM & PM & PM & PM & NM & NM & NM & NM & NM & NM & NM & NM & NM & PM \\
\hline 36 & & NM & NM & NM & NM & NM & NM & NM & NM & NM & NM & NM & NM & NM & NM \\
\hline 37 & & NM & NM & NM & NM & NM & NM & NM & NM & NM & NM & NM & NM & NM & NM \\
\hline 38 & & PM & PM & PM & PM & PM & PM & PM & NM & NM & PM & NM & NM & PM & PM \\
\hline 39 & & $\mathrm{CM}$ & $\mathrm{CM}$ & CM & CM & $\mathrm{CM}$ & $\mathrm{CM}$ & CM & $\mathrm{CM}$ & PM & $\mathrm{CM}$ & $\mathrm{CM}$ & $\mathrm{CM}$ & $\mathrm{CM}$ & $\mathrm{CM}$ \\
\hline 40 & & NM & PM & PM & PM & NM & NM & PM & PM & NM & NM & PM & PM & $\mathbf{P M}$ & $\mathbf{P M}$ \\
\hline 41 & & CM & CM & PN & CM & CM & CM & CM & CM & PM & CM & CM & CM & CM & CM \\
\hline 42 & & CM & CM & CM & CM & $\mathrm{CM}$ & CM & CM & CM & PM & CM & CM & CM & CM & $\mathrm{CM}$ \\
\hline 43 & & PM & $\mathrm{CM}$ & PM & CM & $\mathrm{CM}$ & CM & PM & PM & PM & CM & CM & CM & PM & PM \\
\hline 44 & & NM & NM & NM & NM & NM & NM & NM & NM & NM & NM & NM & NM & NM & NM \\
\hline 45 & & NM & NM & NM & NM & NM & NM & NM & NM & NM & NM & NM & NM & NM & NM \\
\hline 46 & & $\mathrm{CM}$ & $\mathrm{CM}$ & CM & $\mathrm{CM}$ & CM & CM & $\mathrm{CM}$ & $\mathrm{CM}$ & $\mathrm{CM}$ & $\mathrm{CM}$ & CM & $\mathrm{CM}$ & $\mathrm{CM}$ & $\mathrm{CM}$ \\
\hline 47 & & NM & NM & NM & NM & NM & NM & $\mathbf{N M}$ & NM & NM & NM & NM & NM & NM & NM \\
\hline 48 & & $\mathrm{CM}$ & $\mathrm{CM}$ & $\mathrm{CM}$ & $\mathrm{CM}$ & $\mathrm{CM}$ & CM & $\mathbf{C M}$ & $\mathrm{CM}$ & CM & $\mathbf{C M}$ & $\mathrm{CM}$ & $\mathrm{CM}$ & $\mathrm{CM}$ & $\mathrm{CM}$ \\
\hline 49 & & NM & NM & NM & NM & NM & NM & $\mathbf{N M}$ & NM & NM & NM & NM & NM & NM & NM \\
\hline 50 & & NM & $\mathrm{NM}$ & PM & NM & NM & NM & PM & NM & NM & NM & NM & $\mathrm{NM}$ & NM & NM \\
\hline
\end{tabular}

(1) Consensus diagnosis-defined if greater than, or equal to, five pathologists agreeing for both runs

(ii) interobserver agreement- $\mathrm{K}$ value ${ }^{7}$ calculated for each "pair" of pathologists:

$$
\kappa=\frac{\mathrm{Po}-\mathrm{Pe}}{1-\mathrm{Pe}}
$$

where $\mathrm{Po}=$ observed agreement

$\mathrm{Pe}=$ agreement expected by chance values $\quad 1.0=$ perfect agreement

$>0.75=$ excellent agreement beyond chance

$$
\begin{aligned}
& 0.4-0.75= \text { fair to good agreement } \\
& \text { beyond chance } \\
&<0.4=\text { poor agreement beyond } \\
& \text { chance }
\end{aligned}
$$

(iii) Intraobserver agreement-percentage agreement between two runs for each pathologist.

Table 3 Distribution of diagnosis

\begin{tabular}{ll}
\hline Diagnosis & Number of slides with consensus \\
\hline Non-molar pregnancy & 17 \\
Partial mole & 4 \\
Complete mole & 14 \\
\hline
\end{tabular}

\section{Results}

Table 2 shows the answers for each run. Only 35 out of the 50 slides achieved consensus.

Table 3 gives the distribution of these cases. Of the 15 cases not reaching consensus, two were problems of differentiating partial mole from complete mole (cases 18 and 43 ). The

\begin{tabular}{|c|c|c|c|c|c|c|}
\hline & $B$ & $C$ & $D$ & $E$ & $F$ & $G$ \\
\hline $\begin{array}{l}\mathbf{A} \\
\mathbf{B} \\
\mathbf{C} \\
\mathbf{D} \\
\mathbf{E} \\
\mathbf{F}\end{array}$ & .589 & $\begin{array}{r}.690 \\
.491\end{array}$ & $\begin{array}{l}.745 \\
.658 \\
.773\end{array}$ & $\begin{array}{l}.670 \\
.455 \\
.563 \\
.618\end{array}$ & $\begin{array}{r}.586 \\
.417 \\
.578 \\
.628 \\
.466\end{array}$ & $\begin{array}{l}.595 \\
.561 \\
.501 \\
.599 \\
.459 \\
.393\end{array}$ \\
\hline
\end{tabular}
other 13 involved the decision between nonmolar pregnancy and partial mole. There was no problem in differentiating complete mole from non-molar pregnancy.

Table 4 Kappa values

Table 5 Intraobserver agreement

\begin{tabular}{ll}
\hline & Agreement \% \\
\hline A & 84 \\
B & 74 \\
D & 90 \\
E & 72 \\
F & 74 \\
G & 82 \\
\hline
\end{tabular}


Figure 1 Complete mole showing circumferential trophoblast hyperplasia and swollen avascular villi.

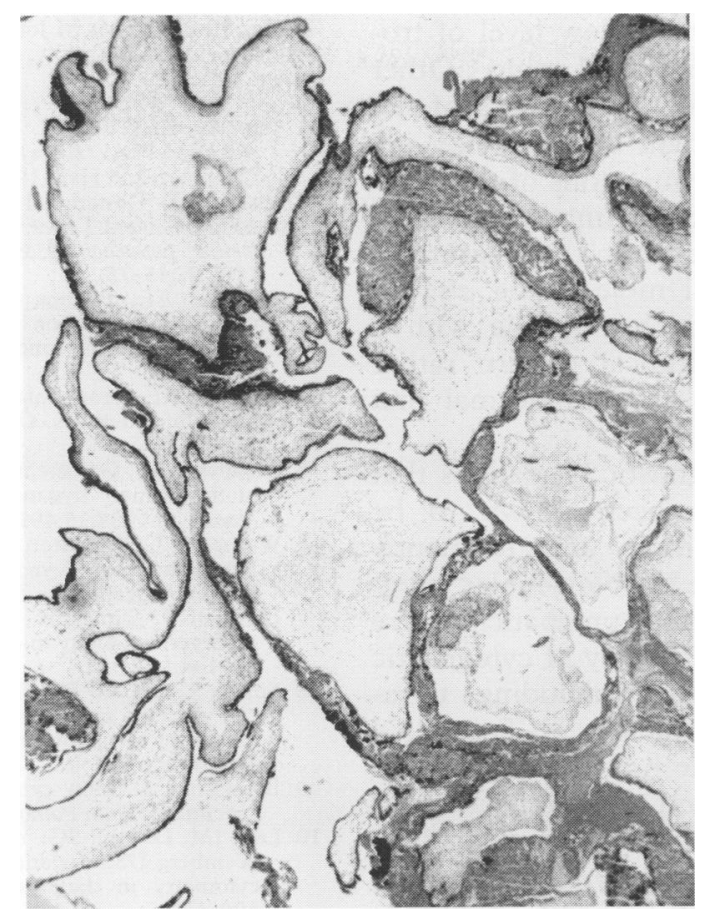

Figure 2 Partial mole showing villi with central cisternal degeneration, scalloping of villi with a "Norvegian fjord" periphery, and mild trophoblastic hyperplasia.

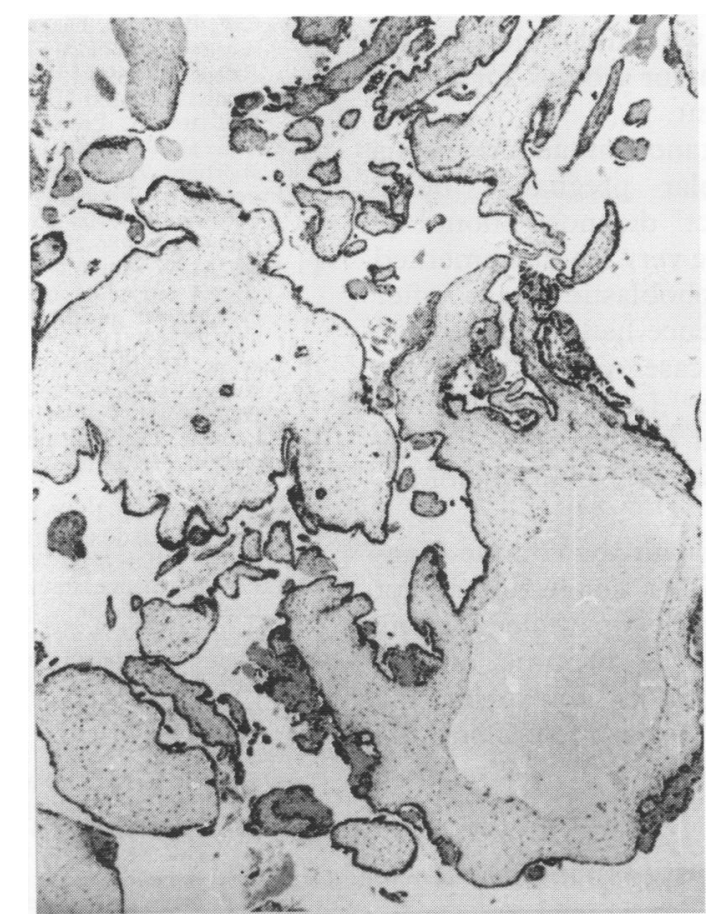

Table 4 shows the $\kappa$ values using data from run 1. Agreement varied between poor (pathologist $\mathrm{F} v \mathrm{G})$ to excellent $(\mathrm{C} v \mathrm{D})$. The values are for all 50 cases, including the 15 cases for which no consensus was established.

Table 5 shows the intraobserver agreement. The values are good to excellent, ranging from $72-90 \%$.

\section{Discussion}

About $15 \%$ of established pregnancies spontaneously abort; dilatation and curettage is often done in these cases to remove any retained products of conception. ${ }^{12}$ When villi and trophoblast are present in the products of

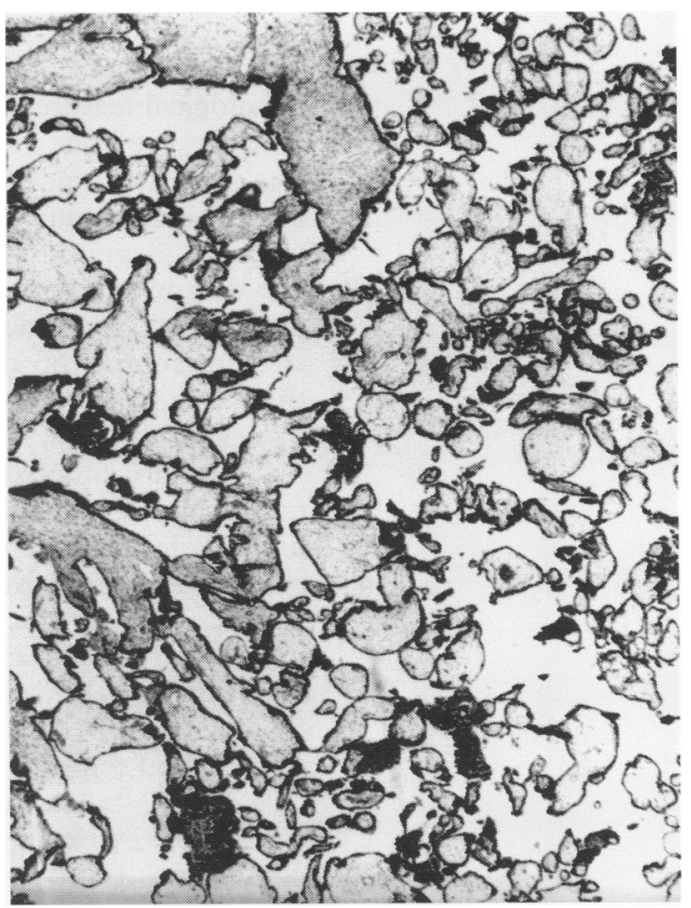

Figure 3 Non-molar pregnancy showing villi with mild hydropic change and no clinically relevant trophoblast hyperplasia.

conception, the pathologist must exclude trophoblastic disease, especially complete mole and partial mole. Complete mole can be reliably distinguished from non-molar pregnancy. In two cases complete mole could not be easily differentiated from partial mole, but this is of little clinical importance as all molar pregnancies should enter the programme for detection of persistent trophoblastic disease. ${ }^{5}$ Unfortunately (but not surprisingly), our study has identified problems differentiating non-molar pregnancy from partial mole. On review, it is clear that many cases of nonmolar pregnancy showed significant hydropic change; the slides were purposely selected to show this feature. Nevertheless, some pathologists would be happy to leave these women and allow them to become pregnant again without follow up; others would impose restrictions on fertility, insisting on urinary $\beta$ human chorionic gonadotrophin follow up. There are extensive histological criteria to avoid this problem (table 1)..$^{1-4}$ Each pathologist seems to feel as though he or she can use these parameters consistently, as shown by the good intraobserver variation (table 5). These comments imply that either the histological criteria for partial mole are not being consistently applied among pathologists or that they are less than ideal for diagnosis. Our collective experience shows that in a nonmolar hydropic pregnancy vesicles are hardly ever seen macroscopically. In partial mole one can see quite large (not small) vesicles mixed with normal villi. There is only mild trophoblast hyperplasia in most cases of partial mole and it is quite incorrect to say that there is hyperplasia of syncytiotrophoblast; syncytiotrophoblast is post-mitotic terminally differentiated tissue, incapable of mitotic activity. Recent studies with proliferating cell 
nuclear antigen support the low level of trophoblast hyperplasia in partial mole. ${ }^{8}$ Other histological features, such as scalloping of villi and the presence of small fibrosed villi, are also seen in surgical terminations of pregnancy and are, in our opinion, unhelpful in differential diagnosis. The important feature in the diagnosis of partial mole is the atypical pattern of trophoblastic hyperplasia with a circumferential or multifocal pattern rather than the polar growth seen in normal first trimester placenta.

There are other diagnostic modalities that may help. Ploidy has been shown to be diploid in complete mole and frequently triploid in partial mole. ${ }^{9-12}$ Non-molar pregnancy, if anembryonic pregnancies are included, shows a wide variety of cytogenetic and ploidy abnormalities including tetraploidy, trisomy, and triploidy. ${ }^{1314}$ Tetraploid and diploid partial mole however, have been described. ${ }^{15} 16$ Assessment of ploidy involves either flow cytometry or static image analysis cytometry, both techniques being mostly unavailable in district general hospitals. Nevertheless, in cases where there is a serious problem in differentiating partial mole from non-molar pregnancy with hydropic change, sending some wet tissue or a block for ploidy studies might be prudent.

What is the importance of an erroneous diagnosis of non-molar pregnancy being made when the "correct" diagnosis should be partial mole? There are very few documented cases of persistent trophoblastic disease after partial mole; the incidence has been reported to vary from 0 of 51 cases partial mole ${ }^{15}$ to eight of 81 partial mole. ${ }^{17}$ Even cases of choriocarcinoma consequent on partial mole have been described. ${ }^{18-21}$ The risk is real, therefore, if very small.

There are problems with the routine diagnosis of partial mole. This conclusion is not novel. ${ }^{22}$ It seems that histopathology alone cannot solve this diagnostic dilemma, but the situation may be helped by improving the diagnostic criteria for partial mole along the lines that we have suggested.

We thank Preston and Chorley Hospitals Research Fund for financial assistance.
Thanks also go to Ms M Jones, medical statistician, Northern Regional Health Authority, Newcastle upon Tyne, for expert statistical help.

1 Buckley $\mathrm{CH}$, Fox $\mathrm{H}$. Biopsy pathology of the endometrium. In: Biopsy pathology series 14. London: metrium. In: Biopsy pathology

2 Elston CW. Gestational trophoblastic disease. In: Fox $\mathrm{H}$ ed. Haines and Taylor. Textbook of obstetrical and gynaeco-
logical pathology. Edinburgh: Churchill Livingstone, logical pathology.

3 Szulman AE, Phillipe E, Boue JG, Boue A. Human triploidy: association with partial hydatidiform moles and non-molar conceptuses. Hum Pathol 1981;12: 1016-21.

4 Szulman AE. Trophoblastic disease: clinical pathology of hydatidiform moles. Obstet Gynecol Clin North Am 1988; 15:443-56.

5 Berkowitz RS, Goldstein DP. Diagnosis and management of the primary hydatidiform mole. Obstet Gynecol Clin North $\mathrm{Am}$ 1988;15:491-503.

6 Womack CC, Elston CW. Hydatidiform mole in Nottingham: a 12 yr retrospective epidemiological and mottingham: a 12 yr retrospective epidemiological study. Placenta 1985;6:93-106.

7 Brennan P, Silman A. Statistical methods for assessing observer variability in clinical measures. $B M \mathscr{F}$ 1992;304: $1491-4$.

8 Suresh UR, Hale RJ, Fox H, Buckley CH. Proliferating cell nuclear antigen (PCNA) immunoreactivity as a means for distinguishing hydropic abortions from partial hydatidiform moles. $f$ Clin Pathol 1993;46:48-50.

9 Benirschke K. Flow cytometry for all mole-like abortion specimens. Hum Pathol 1989;20:403-4.

10 Lage JM, Driscoll SG, Yavner DL, Olivier AP, Mark SD, Weinberg DS. Hydatidiform moles. Application of flow cytometry in diagnosis. Am $f$ Clin Pathol 1988;89: 596-600.

11 Bagshawe $\mathrm{KD}$, Lawler SD. Unmasking moles. $\mathrm{Br} \mathcal{F}$ Obstet Gynaecol 1982;89:255-7.

12 Hemming JD, Quirke P, Womack C, Wells $M$, Elston CW, Bird CC. Diagnosis of molar pregnancy and persistent trophoblastic disease by flow cytometry. $\mathcal{F}$ Clin Pathol 1987;40:615-20.

13 Guerneri S, Bettio D, Simoni G, Brambati B, Lanzani A, Fraccaro $M$. Prevalence and distribution of chromosome abnormalities in a sample of first trimester internal abortions. Hum Reproduct 1987;2:735-9.

14 Procter SE, Watt J, Gray ES. Cytogenetic analysis of 100 spontaneous abortions in North-West Scotland. Clin Genet 1986;29:101-3.

15 Lage JM, Weinberg DS, Yavner DL, Bieber FR. The biology of tetraploid hydatidiform moles: histopathology, cytogenetics and flow cytometry. Hum Pathol 1989;20 419-25.

16 Lawler SD, Fisher RA, Dent J. A prospective genetic study of complete and partial hydatidiform moles. $A m \mathcal{F}$ Obstet Gynecol 1991;164:1270-7.

17 Berkowitz RS, Goldstein DP, Bernstein MR. Natural history of partial molar pregnancy. Obstet Gynecol 1986; 66:677-81.

18 Gardner HAR, Lage JM. Choriocarcinoma following a partial hydatidiform mole: a case report. Hum Patho 1992;23:468-71.

19 Looi LM, Siganesaratnam V. Malignant evolutions with fatal outcome in a patient with partial hydatidiform fatal outcome in a patient with partial hydatic
mole. Aust NZ $\mathcal{Y}$ Obstet Gynaecol 1981;21:51-2.

20 Heifetz SA, Czaja J. In situ choriocarcinoma arising in partial hydatidiform mole: implications for the risk of persistent trophoblastic disease. Paediatr Pathol 1992;12 601-11.

21 Bagshawe KD, Lawler SD, Paradinas FJ, Dent J, Brown $\mathrm{P}$, Boxer GM. Gestational trophoblastic tumours following initial diagnosis of partial hydatidiform mole. Lancet 1990;335:1074-6.

22 Javey H, Borazjani G, Behmard S, Langley FA. Discrepancies in the histological diagnosis of hydatidiform mole. Br F Obstet Gynaecol 1979;86:480-3. 